\title{
RECONFIGURABLE MODELLING WITH DEADLOCK AVOIDANCE FOR THE CLINICAL PATHWAY BASED ON MCPN-CS
}

\author{
Du, G. ; Jiang, Z. B. ; Diao, X. D. ${ }^{* *} \&$ Yao, Y.** \\ * Department of Industrial Engineering and Logistics Management, Shanghai Jiao Tong University, \\ Shanghai 200240, China \\ ** Shanghai Putuo District Central Hospital, Shanghai 200062, China \\ ${ }^{* * *}$ Shanghai No. 6 People’s Hospital, Shanghai 200233, China \\ E-Mail: dg_001@sjtu.edu.cn, zbjiang@sjtu.edu.cn
}

\begin{abstract}
Clinical Pathway (CP) is very complicated and has many exceptional variations. Generally, its treatment course and control steps cannot be totally predefined. Meanwhile, the CP embodies the "Re-flow" therapy features, which is very hard to model, control and manage. Therefore, combined modular modelling method and structure changing mechanisms, a Modular Coloured Petri Net with changeable structure (MCPN-CS) workflow modelling method is proposed. Aimed at the variations of the $\mathrm{CP}$, the workflow model for the $\mathrm{CP}$ can be reconfigured dynamically by using the mechanisms of change-by-modification (CBM) and change-by-composition (CBC). A case study on a workflow modelling of osteosarcoma CP is constructed and the modelling is analyzed by proposed deadlock detection algorithms (DDA). The result validates that the proposed method may noticeably enhance the flexibility, adaptation, reusability and maintainability of the workflow model for the CP.

(Received in March 2009, accepted in January 2010. This paper was with the authors 2 months for 1 revision.)
\end{abstract}

Key Words: Clinical Pathway (CP), Workflow Modelling, MCPN-CS, Changeable Structure, Deadlock Detection

\section{INTRODUCTION}

Clinical pathway (CP) is a management plan that displays goals for patients and provides the sequence and timing of actions necessary to achieve these goals with optimal efficiency [1, 2]. After being introduced by Zander into medical care in early 1980s, it has been widely applied in hospitals and medical care communities $[3,4]$. Most of references demonstrate that the CP has many advantages for the application in hospitals, like reducing medical expense, improving healthcare service quality and level, etc [5]. The CP, as a new medical management mode, has the following main characteristics: (1) It is very complex for having strong "Re-flow" features, which means that a patient may visit the same therapy phase many times in the whole treatment process; (2) Although the CP has regulated the predictable standard treatment process of certain illness, some unavoidable variations (which considered as any deviations from standardized pathway, such as infection and poisoning) still occur during the execution process usually. It is a very difficult to find a good method for dealing with the variations validly; (3) Complicated logic relations and timely constraints in different treatment activities need to be described precisely. Especially, at present, there have been a lot of reports on the CP implementations, mostly in the paper-based manual way. The predefined paper CP document only presents expected outcomes to be reached and standardized interventions to be performed across a daily timeline. Therefore, it gives rise to many problems. Workflow is the automation of a business process, in whole or part, during which 
documents, information or tasks are passed from one participant to another for action, according to a set of procedural rules [6]. Therefore, the workflow management can automatically coordinate activities in business procedures and thus provides crucial technological means for the CP computerization and automated implementation [7]. Moreover, workflow applications in the healthcare domain have been reported in [8, 9, 10]. However, the traditional workflow management technology only considers and executes the predefined business process. Thus, it is weak in fully supporting the modelling and execution of the CP with "Re-flow" and variations. All these motivate the authors to find a new approach for workflow modelling of the CP.

Petri net, as a state-based description, has been broadly used for workflow modelling [11]. Aalst proposed the formal Workflow-net (WF-net) method for workflow modelling based on a basic Petri net [12]. However, the basic Petri nets describing a real system tend to be complex and extremely large so that extended Petri nets (such as coloured Petri net and hierarchical Petri net) have been used to model a system [13, 14]. Liu et al. proposed a workflow net based on a coloured Petri net (WFCP-net) that is extended with colour from the WF-net [15]. References of research on applying the Modular CPN-based method for modelling production system and workflow system can be found [16]. However, the above methods can not solve the workflow modelling problems of the CP with many uncertain variations. Therefore, a Modular Coloured Petri net with changeable structure (MCPN-CS) modelling method is proposed in this paper. It can be used to solve the "hard" problem from the following two aspects: Firstly, the structure change mechanism can be used to describe the uncertainty and variance of the CP. It includes two types: 1) change-by-modification (CBM); 2) change-by-composition (CBC). The CBM can be used for modification of the structure of current CPN model while CBC can be used for the composition of another CPN into the current CPN [17].

The remainder of this paper is organized as follows: The definition of MCPN-CS is given in Section 2. To illustrate the detailed modelling and deadlock detection procedure, a case study on the osteosarcoma CP is demonstrated in Section 3. Finally, conclusions are drawn in Section 4.

\section{THE DEFINITION OF WORKFLOW MODELLING METHOD BASED ON MCPN-CS}

\subsection{Definition of the MCPN-CS}

The MCPN-CS is a modular structure which is composed of one or more CPN-CSMs. Each CPN-CSM, in turn, encapsulates a Coloured Petri Net with Changeable Structure (CPN-CS) $[18,19,20]$. A CPN-CSM contains a CPN-CS and those interfacing features for communication with the outside world. Each CPN-CSM is encapsulated in a standard container, which is equipped with standard interface. For this, it can be called a Coloured Petri net with Changeable Structure module (CPN-CSM). A CPN-CSM is defined as:

Where:

$$
C P N-C S M_{k, m}=\left(\operatorname{Str}_{k, m}, M_{k, 0, m,} M_{k-1, c, m}, \text { Port }_{k, m}\right)
$$

(1) $C P N-C S M_{k, m}$ denotes the $m^{\text {th }}$ module after the $k^{\text {th }}$ change. $S t r_{k, m}=\left\{\sum_{k, m}, P_{k, m}, T_{k, m}\right.$, $\left.A_{k, m}, C_{k, m}, G_{k, m}, E_{k, m}\right\}$ is the inner structure of the $m^{\text {th }}$ module after the $k^{\text {th }}$ change. Where $\sum_{k, m}, P_{k, m}, T_{k, m}, A_{k, m}, C_{k, m}, G_{k, m}, E_{k, m}$ can are as defined as the $k^{\text {th }}$ change of $\mathrm{CPN}$. When $\mathrm{k}=0$, we obtain the initial structure denoted by $\mathrm{Str}_{0, m}$.

(2) $M_{k, 0, m}$ is the initial marking of the $m^{\text {th }}$ module after the $k^{\text {th }}$ change. $M_{k, 0, m}$ replaces initialization function $I_{k, m}$. 
(3) $M_{k-1, c, m}$ is the marking set of all places of the $m^{\text {th }}$ module just before the $k^{\text {th }}$ change occurs.

(4) Port $_{k, m}$ is the set of ports of the $m^{\text {th }}$ module after the $k^{\text {th }}$ change. Port ${ }_{k, m} s$ act as the information interface between the module and external environment. Information is passed through the ports in the form of coloured tokens.

(a) $i p_{k, m}^{j s}$ is the input port of the $m^{\text {th }}$ module after the $k^{\text {th }}$ change, where $s=1$, $2, \ldots S$, and $S$ is the total number of input ports; $j$ denotes the name of module.

(b) $o p^{j v}{ }_{k, m}$ is the output port of the $m^{\text {th }}$ module after the $k^{\text {th }}$ change, where $v=1$, $2, \ldots V$, and $V$ is the total number of output ports; $j$ denotes the name of module.

Finally, different $C P N-C S M_{k, m} S$ are linked together as a system model by defining the relationship of module. The result of the complex graph of $C P N-C S M$ can be called a $M C P N-C S$, which is defined as:

$$
M C P N-C S=\left(C P N-C S M_{k, m} S, M B G_{t, n}\right)
$$

The $C P N-C S M_{k, m}$ above are the set of all disjointed $C P N-C S M$ in the model. $M B G_{t, n}$ is a special type of transition linking all disjointed $C P N-C S M_{k, m}$, which are described in Section 2.2 .

\subsection{The definition of Module Interconnection Relations by MBG}

To cope with treatment complexity and "Re-flow" of CP, a special type of transition, namely "Main-Bus Gate" (MBG) [21], is developed to connect all modules in the CP. It can be described as follows:

$$
M B G_{t, n}=\left(\operatorname{StrMBG} G_{t, n}, M_{t, 0, n,} M_{t-1, c, n}\right),
$$

where $M B G_{t, n}$ denotes the $n^{\text {th }}$ kind CP after the $t^{\text {th }}$ change.

StrMBG $G_{t, n}=\left\{P S_{t, n}, S T_{t, n}, I n S_{t, n}\right.$, Out $\left._{t, n}\right\}$ is the structure of the MBG of the $n^{\text {th }}$ kind CP after the $t^{\text {th }}$ change. When $t=0$, we obtain the initial structure denoted by $\operatorname{Str} M B G_{0, n} P S_{t, n}, S T_{t, n}$, InS $S_{t, n}$, Out $S_{t, n}$ can be described as follows:

(1) $P S_{t, n}=\left\{P S_{t, n}^{i}, i=1,2, \ldots, I\right\}$ is a set of treating stages of a patient that he or she has to undergo in CP of the $n^{\text {th }}$ kind CP after the $t^{\text {th }}$ change, where $i$ is the index of treating stages.

(2) $S T_{t, n}=\left\{S T_{t, n}^{j}, j=1,2, \ldots, J\right\}$ is a set of modules of the $n^{\text {th }}$ kind CP after the $t^{\text {th }}$ change, where $\mathrm{J}$ is the total number of modules in CP.

where:

$$
S T_{t, n}^{j}=\left\{\left[i p_{k, m}^{j s}\left(c t_{L, j}\right)>o p^{j v}{ }_{k, m}\left(c t_{L, i+1}\right)\right], i=1,2, \ldots I\right\},
$$

(a) The definitions of $i p^{j s}{ }_{k, m}$ and $o p^{j v}{ }_{k, m}$ can be seen in Section 2.1;

(b) $c t_{i}$ and $c t_{i+1}$ are the coloured tokens representing a patient that wait for treatment in module $j$ and the one that completes treating in module $j$ respectively; $i$ denotes the treatment step;

(c) ' $>$ ' denotes if-then;

(3) $I n S_{t, n}=o p_{t, n}^{P E}$ is the output port of "Physical examination (PE)" module of the $n^{\text {th }}$ kind $\mathrm{CP}$ after the $t^{\text {th }}$ change.

(4) $O u t S_{t, n}=i p_{t, n}^{M F V}$ is the input port of "Makes a follow up visit (MFV)" module of the $n^{\text {th }}$ kind CP after the $t^{\text {th }}$ change.

(5) $M_{t, 0, \mathrm{n}}$ is the initial marking of MBG of the $n^{\text {th }}$ kind CP after the $t^{\text {th }}$ change.

(6) $M_{t-1, c, n}$ is the marking set of all places in MBG just before the $t^{\text {th }}$ change of the $n^{\text {th }}$ kind CP occurs, where $M B G_{t, n}$ is the MBG before the $n^{\text {th }}$ kind CP after the $t^{\text {th }}$ change. 


\section{DEVELOPMENT AND ANALYSIS OF WORKFLOW MODEL FOR THE CP BASED ON MCPN-CS}

Aimed at the characters of the CP, the development and analysis of the MCPN-CS model process include 5 phases: 1) Building the CPN-CSM for individual treatment module in the system; 2) Integrating each module to construct the whole system model by the MBG; 3) Deadlock detection of each module and the whole system model as well; 4) According to the variations of the $\mathrm{CP}$, the $\mathrm{CP}$ model is reconfigured by CBM, CBC or CBM+CBC mechanism. The deadlock detection of the model after reconfiguration is analyzed; 5) The reconfiguration of the whole CP workflow model and the deadlock detection of the whole system are illustrated. An example is taken to illustrate the procedure of the MCPN-CS-based workflow modelling of the stage-IIB osteosarcoma CP. Suppose the osteosarcoma patient enters into the CP. The entire process contains 5 main modules: "Diagnosis", "Staging", "Preoperative Chemotherapy", "Operation", "Postoperative Evaluation and Chemotherapy" and two input/output modules: "Physical Examination”, and "Makes a Follow up Visit”.

\subsection{Building the CPN-CSM for individual treatment module}

In this section, only the preoperative chemotherapy module (because of variations occur usually in this module) is given to illustrate how to build the CPN-CSM model. Places of activities represent the events and change of conditions from one activity to another. The model of preoperative chemotherapy module can be seen in Fig. 1 and Table I. The "ip ${ }_{0,3}^{P R C 01}$ " indicates input information of "Chemotherapy Requesting", and the " $o p_{0,3}^{P R E C 01}$ indicates output information of "Preoperative Chemotherapy finished”.

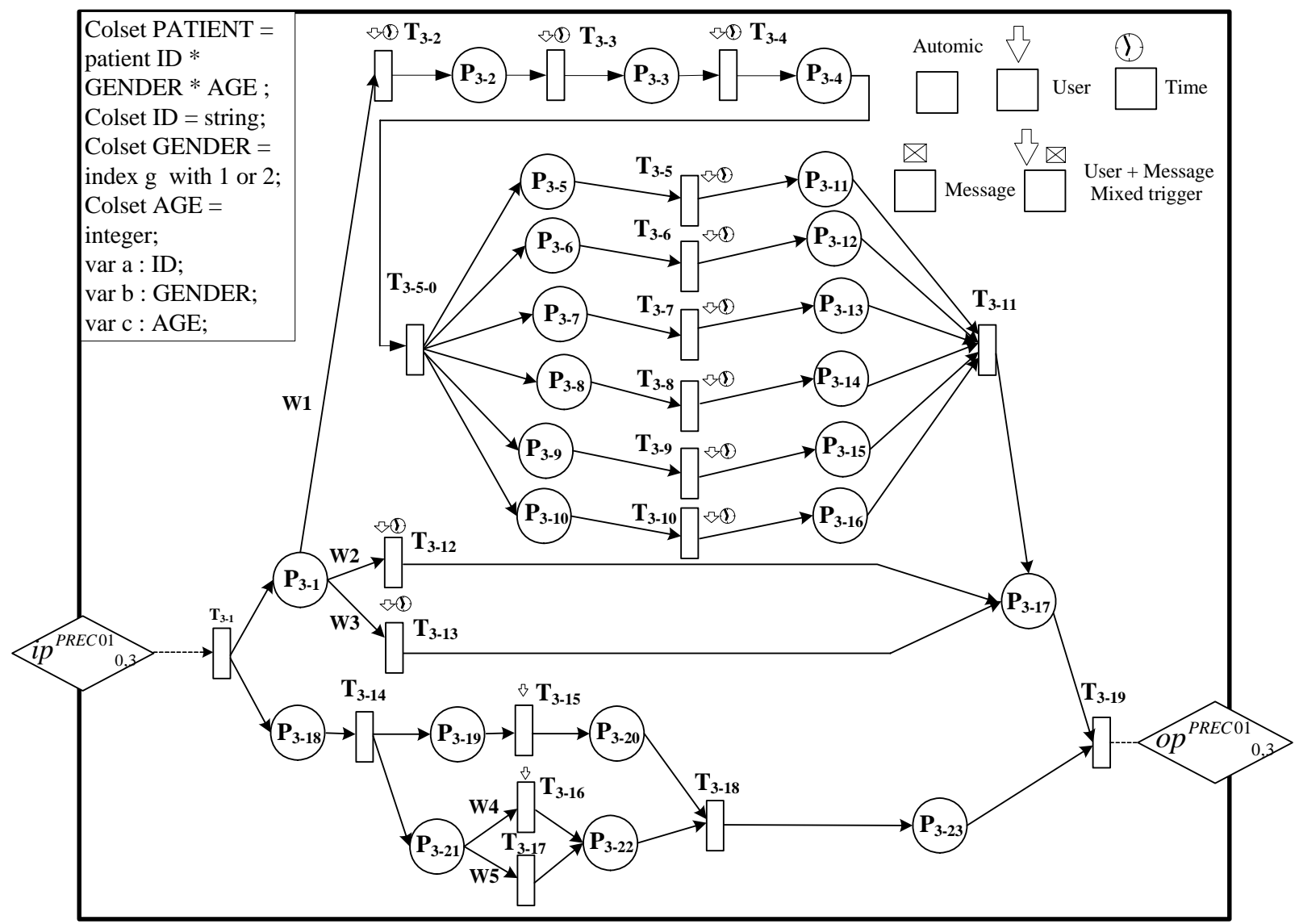

Figure 1: The structure of the MCPN-CS-based preoperative chemotherapy. 
Du, Jiang, Diao, Yao: Reconfigurable Modelling with Deadlock Avoidance for the Clinical ...

Table I: Transition definition table of the preoperative chemotherapy module.

\begin{tabular}{|c|l|c|l|}
\hline Transition & \multicolumn{1}{|c|}{ Description } & Transition & \multicolumn{1}{|c|}{ Description } \\
\hline T3-1 & $\begin{array}{l}\text { Preparation of chemotherapy is } \\
\text { processing }\end{array}$ & $\mathrm{T}_{3-10}$ & Skin checking \\
\hline $\mathrm{T}_{3-2}$ & $\begin{array}{l}\text { Ready for the treatment scheme of } \\
\text { high-dose MTX and CF rescue }\end{array}$ & $\mathrm{T}_{3-11}$ & $\begin{array}{l}\text { The checking results of } \\
\text { blood, liver and kidney } \\
\text { function are collected }\end{array}$ \\
\hline $\mathrm{T}_{3-3}$ & $\begin{array}{l}\text { High-dose MTX and CF rescue are } \\
\text { used }\end{array}$ & $\mathrm{T}_{3-12}$ & HIRP therapy \\
\hline $\mathrm{T}_{3-4}$ & $\begin{array}{l}\text { Monitoring of urine, blood, liver and } \\
\text { kidney function, MTX concentration }\end{array}$ & $\mathrm{T}_{3-13}$ & Arterial chemotherapy \\
\hline $\mathrm{T}_{3-5-0}$ & $\begin{array}{l}\text { Beginning to prepare for the checking } \\
\text { of Blood routine, liver and kidney } \\
\text { function }\end{array}$ & $\mathrm{T}_{3-14}$ & $\begin{array}{l}\text { Preparation of osteosarcoma } \\
\text { assistant therapy is processing }\end{array}$ \\
\hline $\mathrm{T}_{3-5}$ & $\begin{array}{l}\text { Beginning to prepare for the Blood } \\
\text { routine checking }\end{array}$ & $\mathrm{T}_{3-15}$ & $\begin{array}{l}\text { Stopping vomit and filling } \\
\text { medicine generally therapy }\end{array}$ \\
\hline $\mathrm{T}_{3-6}$ & Kidney function checking & $\mathrm{T}_{3-16}$ & Nutrition therapy \\
\hline $\mathrm{T}_{3-7}$ & $\begin{array}{l}\text { Renal function and Uronoscopy } \\
\text { checking }\end{array}$ & $\mathrm{T}_{3-17}$ & Dumb \\
\hline $\mathrm{T}_{3-8}$ & Nonnasality checking & $\mathrm{T}_{3-18}$ & Nutrition-supporting therapy \\
\hline $\mathrm{T}_{3-9}$ & Gastrointestinal checking & $\mathrm{T}_{3-19}$ & Summarizing chemotherapy \\
\hline
\end{tabular}

\subsection{Integrating individual model to construct the whole system model by MBG}

The whole system model includes many treatment modules and two input/output modules. The model can be integrated by MBG, which is developed based on the roughly Treatment Sequence Definition (TSD) and corresponding Patient-Clinical Pathway Matrix (PCPM). Because TSD builds a linkage between patients and modules, every therapy step of the patient is related to a certain module. The content of a patient's TSD in the process of CP can be transferred into a matrix form through the Patient-CP mapping, which is called "Patient-Clinical Pathway Matrix (PCPM)".

Table II: PCPM illustration of the II A stage of the osteosarcoma CP.

\begin{tabular}{|l|l|l|l|l|l|l|}
\hline & $\ldots$ & DG & SG & PREC & EMSS & ETP \\
\hline$\ldots \ldots$ & $\ldots$ & $\ldots$ & $\ldots$ & $\ldots$ & $\ldots$ & $\ldots$ \\
\hline DGSG & $\ldots$ & $3 / 8$ & 4 & $/$ & $/$ & $\ldots$ \\
\hline PRET & $\ldots$ & $/$ & $/$ & 5 & $/$ & $\ldots$ \\
\hline EMSSOP & $\ldots$ & $/$ & $/$ & $/$ & 6 & $\ldots$ \\
\hline POST & $\ldots$ & $\ldots$ & $\ldots$ & $\ldots$ & $\ldots$ & $7 / 9$ \\
\hline
\end{tabular}

/: No treating operations

As shown in Table II, PCPM's column shows the phase of patients' diagnoses and treatments, where the therapy sequence is from left to right and its row indicates the modules in the CP. The table determines the sequence of the therapy and their corresponding modules. In this example, when the patient undergoes its "DGSG" treating stage, its $3^{\text {rd }}$ step is treated in the "DIAGNOSIS (DG)" module, and its $4^{\text {th }}$ step will be treated in the "STAGING (SG)" module, and its $7^{\text {th }}$ step will be treated in the "EVALUATION AND TREATMENT OF 
POSTOPERATION (ETP)" module, then the patient will return "DIAGNOSIS" module to fulfil its $8^{\text {th }}$ therapy step. When the patient enters into CP, its PCPM list of corresponding treatment stage will be loaded into the MBG dynamically.

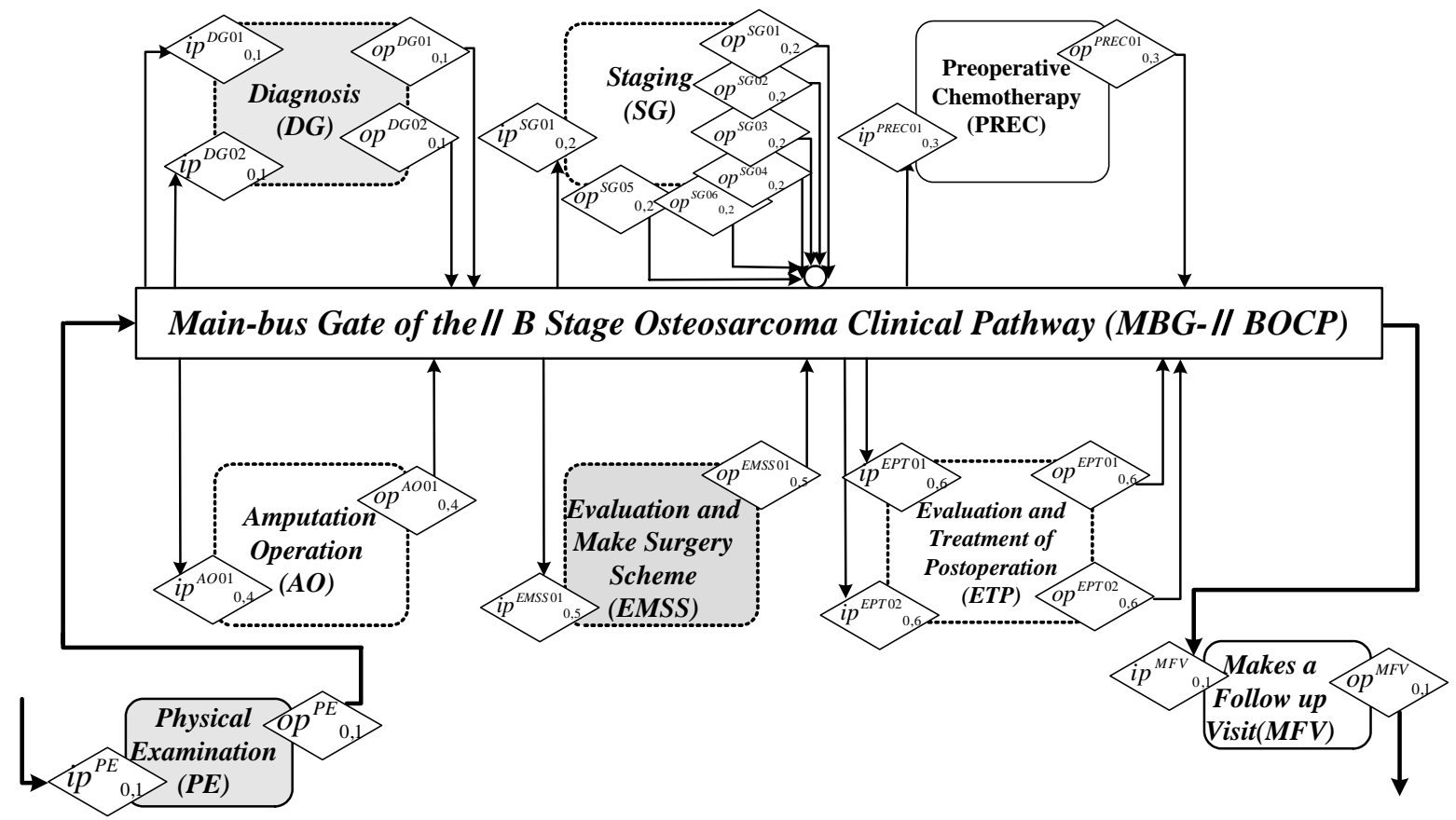

Figure 2: MCPN-CS model of the II B stage osteosarcoma CP.

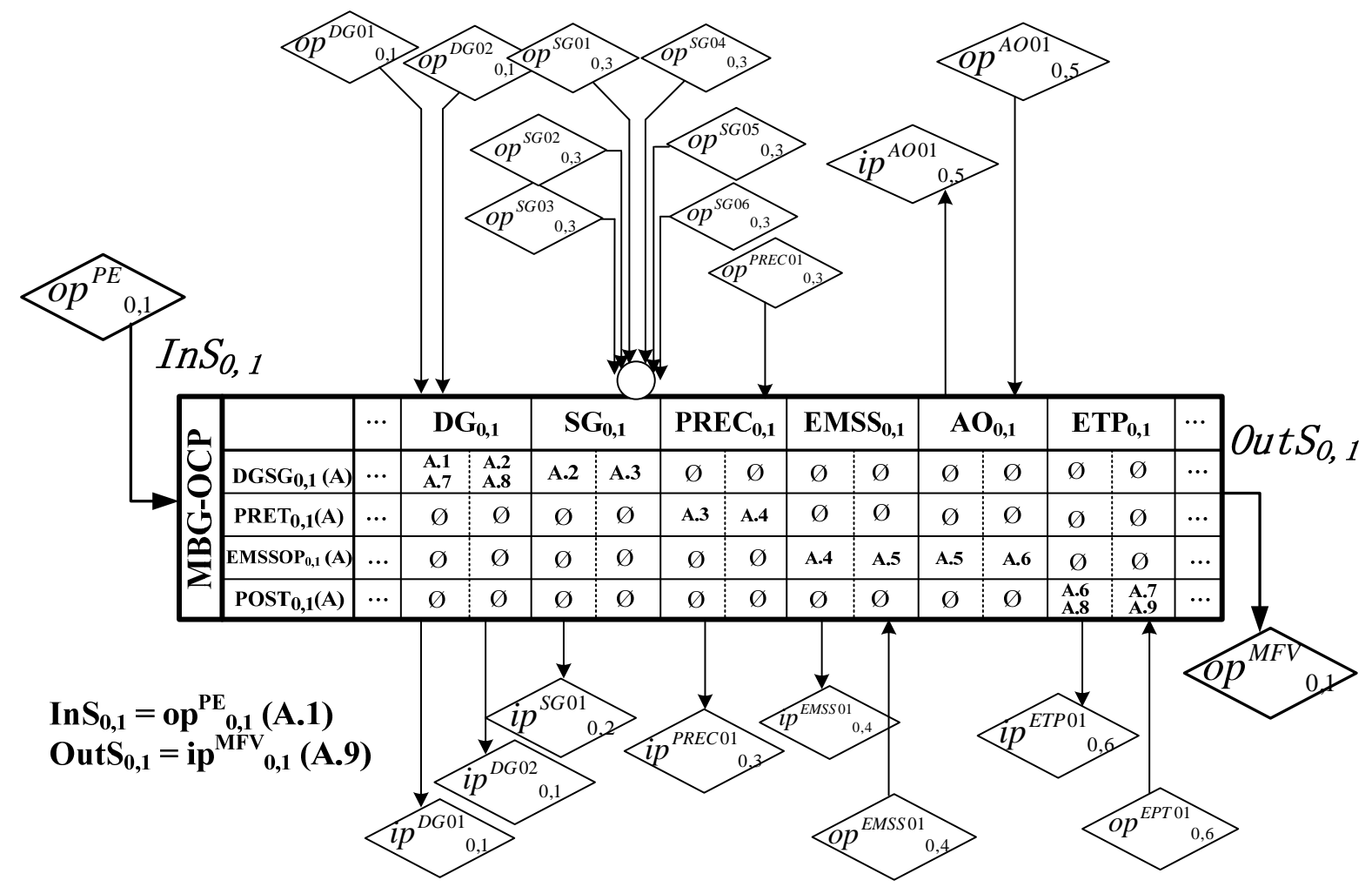

Figure 3: The MBG of the II B stage osteosarcoma CP.

After the last step of this stage is completed, the information and its PCPM list will also be cleared out of the MBG. Therefore, the MBG may lead every patient to precisely execute 
their treatment steps. Detailed example is shown in Fig. 2. Fig. 3 gives the MBG of the II B stage osteosarcoma CP. For instance, patient A.1 undergoes the DGSG treating stage (DGSG (A)), and this stage includes 2 treating steps. After having been treated in the "DG" module for A.1, it will turn into A.2, and then it will go to 'SG' module to undergo its $2^{\text {nd }}$ treating step. After that, a coloured token A.3 will be removed from the CP by output module, and its PCPM list "DGSG (A)" will be removed from the MBG.

\subsection{Deadlock detection of each module and the whole system model}

In this paper, we concentrate on the deadlock analysis of the whole MCPN-CS model connected by MBG. The Deadlock Detection Algorithm (DDA), derived from [22] will be enhanced and applied. To implement the DDA, the MBG-connected CPN-CSM model shown in Fig. 3 must be transformed to a form that is composed of a set of ordinary places and transitions. Formal transformation procedure and deadlock analysis by using DDA can be seen in [21]. By this method, the transformed MCPN-CS model of the II B stage of osteosarcoma CP can be seen in Fig. 4, and the token will be fired by the sequence of the patient treatment.

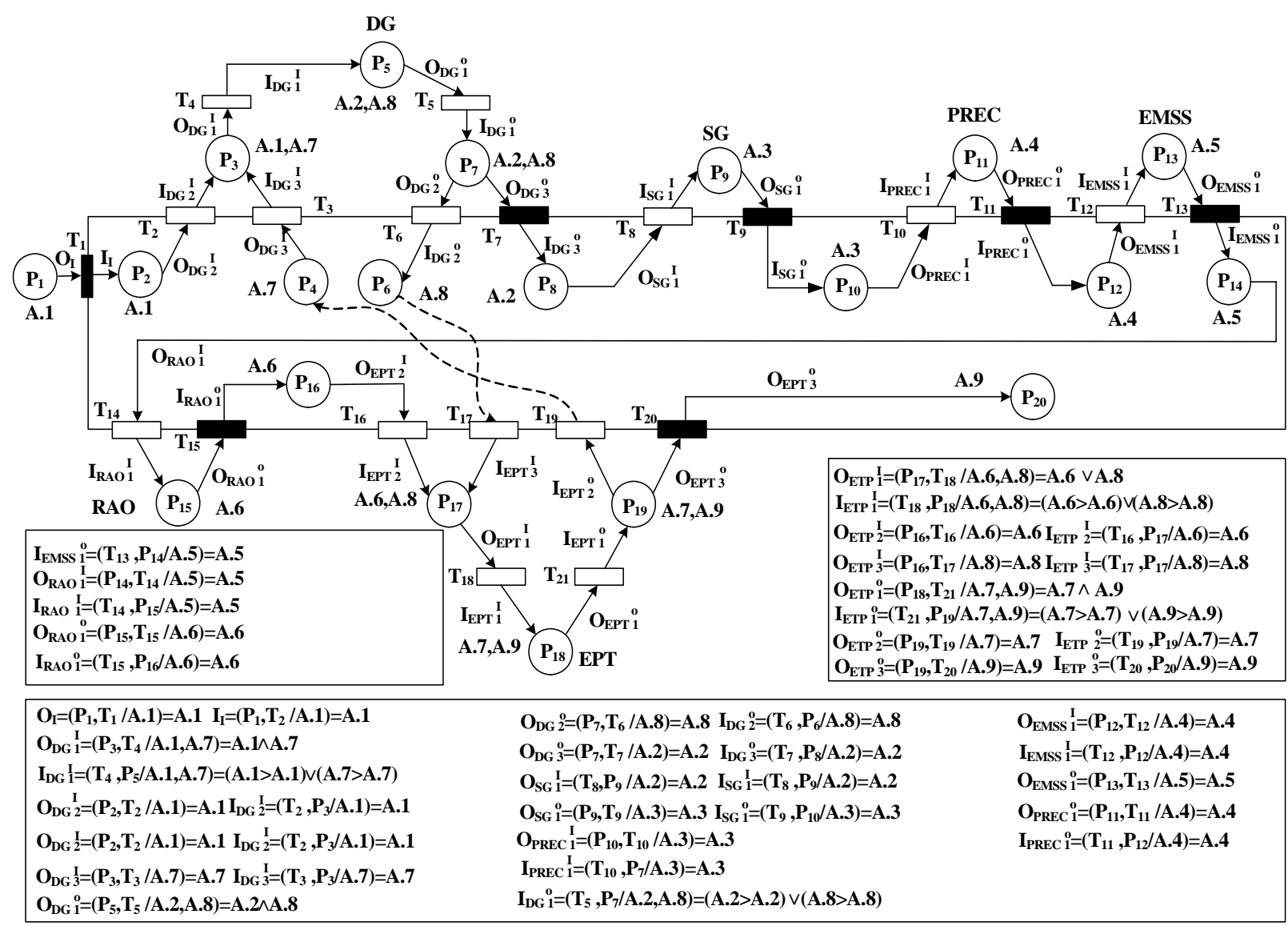

Figure 4: The transformed MCPN-CS model of the II B stage osteosarcoma CP.

Suppose the initial state $\mathrm{M}_{0}$ and the goal state $\mathrm{M}_{\mathrm{g}}$ can be listed as follows:

$$
\begin{aligned}
& M_{0}=(A .1,0,0,0,0,0,0,0,0,0,0,0,0,0,0,0,0,0,0,0,0) \\
& M_{g}=(0,0,0,0,0,0,0,0,0,0,0,0,0,0,0,0,0,0,0,0, A .9)
\end{aligned}
$$

A transformed gate $G_{j}$, including the MIG/MOG and SIG/SOG, associated with a coloured token $c t$ is enabled if $M\left(M P_{k}\right) \geq \mathrm{O}\left(M P_{k}, G_{j} c t\right)$. When $k=0, T_{\text {enable-k }}=\left\{\left(P_{1}, T_{1} / A .1\right)\right\}=\{$ A.1 $\}$. Since 
$M\left(P_{1}\right)=(A .1) \geq\{A .1\},\left(T_{1} /(A .1)\right)$ may be fired with the coloured tokens $A .1$ which will be moved to $\mathrm{P}_{2}$. So the characteristic vector can be determined as:

$$
S=(\text { A.1, } 0,0,0,0,0,0,0,0,0,0,0,0,0,0,0,0,0,0,0,0)^{-1} .
$$

The resulting marking of the next state:

$$
\begin{aligned}
M_{1}= & (0, A .1,0,0,0,0,0,0,0,0,0,0,0,0,0,0,0,0,0,0,0) \neq M_{g} \text {, we need to set } k=k+1 . \\
M_{2}^{-1} & =M_{1}{ }^{-1}+A \times S=M_{1}^{-1}+A \times(A .1,0,0,0,0,0,0,0,0,0,0,0,0,0,0,0,0,0,0,0,0)^{-1} \\
& =(0,0, A .1,0,0,0,0,0,0,0,0,0,0,0,0,0,0,0,0,0,0)^{-1}
\end{aligned}
$$

Therefore, $M_{2} \neq M_{g}$.

In the same way, DDA can find out whether the MCPN-CS model is live and deadlockfree. After analysis, the MCPN-CS model depicted in Fig. 3 is validated live and deadlockfree.

\subsection{Structural changes of MCPN-CS-based Workflow model of the osteosarcoma CP}

By MCPN-CS method, taking “osteosarcoma preoperative chemotherapy” module for example, the execution of the CP can be simulated or controlled. By the simulation, we may find executable occurrence sequence of transitions. A possible occurrence sequence leading to this marking is shown as follows:

$M_{0,0,3}\left[\left(Y_{0,1,3}, Y_{0,2,3}, Y_{0,3,3}, Y_{0,4,3}, Y_{0,5,3}, Y_{0,6,3}, Y_{0,7,3}, Y_{0,8,3}, Y_{0,9,3}, Y_{0,10,3}, Y_{0,11,3}, Y_{0,12,3}, Y_{0,13,3}, Y_{0,14,3}\right.\right.$, $\left.Y_{0,15,3)}\right]>\mathrm{M}_{0,15,3}=\mathrm{M}_{0, \mathrm{c}, 3}$

where $Y_{0,1,3}, Y_{0,2,3}, \ldots, Y_{0,15,3}$ is the occurrence sequence that leads from $M_{0,0,3}$ to $M_{0,15,3}$. $M_{0,15,3}=\left(p_{3-11}, P_{1}\right)+\left(p_{3-12}, P_{1}\right)+\ldots$ is the marking representing that the checking of blood, liver and kidney function have been completed. This occurrence sequence includes 15 steps. A step $Y$ is specified by an enabled transition and binding of its variables. The binding is to evaluate all variables of arc expression functions and guard functions. In this model, there is a patient $P_{1}$. Steps in the occurrence sequence are:

$Y_{0,1,3}=\left(t_{3-1},<P_{1}>\right), Y_{0,2,3}=\left(t_{3-2},<P_{1}>\right), Y_{0,3,3}=\left(t_{3-3}, \quad<P_{1}>\right), Y_{0,4,3}=\left(t_{3-4},<P_{1}>\right), \quad Y_{0,5,3}=\left(t_{3-5-0}\right.$, $\left.<P_{1}>\right), \quad Y_{0,6,3}=\left(t_{3-5}, \quad<P_{1}>\right), \quad Y_{0,7,3}=\left(t_{3-6}, \quad<P_{1}>\right), \quad Y_{0,8,3}=\left(t_{3-7}, \quad<P_{1}>\right), \quad Y_{0,9,3}=\left(t_{3-8},<P_{1}>\right)$,

\begin{tabular}{|c|c|c|c|}
\hline Place & Description & Transition & Description \\
\hline$P_{3-1-1}$ & $\begin{array}{l}\text { Myelosuppression } \\
\text { disappear }\end{array}$ & $\mathrm{T}_{3-1-1}$ & Adding the activity of injection G-CSF \\
\hline$P_{3-1-2}$ & $\begin{array}{l}\text { Kidney function is } \\
\text { normal }\end{array}$ & $\mathrm{T}_{3-1-2}$ & Liver Ganlixin intravenous is injected \\
\hline$P_{3-1-3}$ & $\begin{array}{l}\text { Renal function and } \\
\text { uronoscopy are } \\
\text { normal }\end{array}$ & $\mathrm{T}_{3-1-3}$ & $\begin{array}{c}\text { Chemotherapy is stopped and sodium } \\
\text { bicarbonate of } 150 \mathrm{mg} \text { is added to maintain } \\
\text { urine } \mathrm{pH}>7\end{array}$ \\
\hline $\mathrm{P}_{3-1-4}$ & Stomatitis disappear & $\mathrm{T}_{3-1-4}$ & $\begin{array}{l}\text { The oral ulcer ointment and CF solution are } \\
\text { applied }\end{array}$ \\
\hline$P_{3-1-5}$ & $\begin{array}{l}\text { Gastrointestinal } \\
\text { reactions disappear }\end{array}$ & $\mathrm{T}_{3-1-5}$ & The ondansetron hydrochloride is injected \\
\hline$P_{3-1-6}$ & Skin rash disappear & $\mathrm{T}_{3-1-6}$ & $\begin{array}{l}\text { The hydrocortisone, high-dose intravenous } \\
\text { glucose and sodium chloride are injected }\end{array}$ \\
\hline
\end{tabular}
$Y_{0,10,3}=\left(t_{3-9}, \quad<P_{1}>\right), \quad Y_{0,11,3}=\left(t_{3-10}, \quad<P_{1}>\right), \quad Y_{0,12,3}=\left(t_{3-14}, \quad<P_{1}>\right), \quad Y_{0,13,3}=\left(t_{3-15}, \quad<P_{1}>\right)$, $Y_{0,14,3}=\left(t_{3-16},<P_{1}>\right), Y_{0,15,3}=\left(t_{3-18},<P_{1}>\right)$

Table III: The added places and transitions of “HDMTX+CF” chemotherapy scheme. 
Suppose the following variations of the patient $P_{1}$ appear in osteosarcoma preoperative chemotherapy: 1) Skin rash; 2) Stomatitis; 3) Gastrointestinal reactions; 4) Liver damage; 5) Renal damage; 6) Myelosuppression. Therefore, the workflow modelling of osteosarcoma preoperative chemotherapy needs to be modified $124 \mathrm{~h}$ after the chemotherapy starts. To model this change to the osteosarcoma preoperative chemotherapy $\mathrm{CP}$, the interrelated control measures should be used. By CBM algorithm, the changed MCPN-CS model is shown in Fig. 5 and Table III.

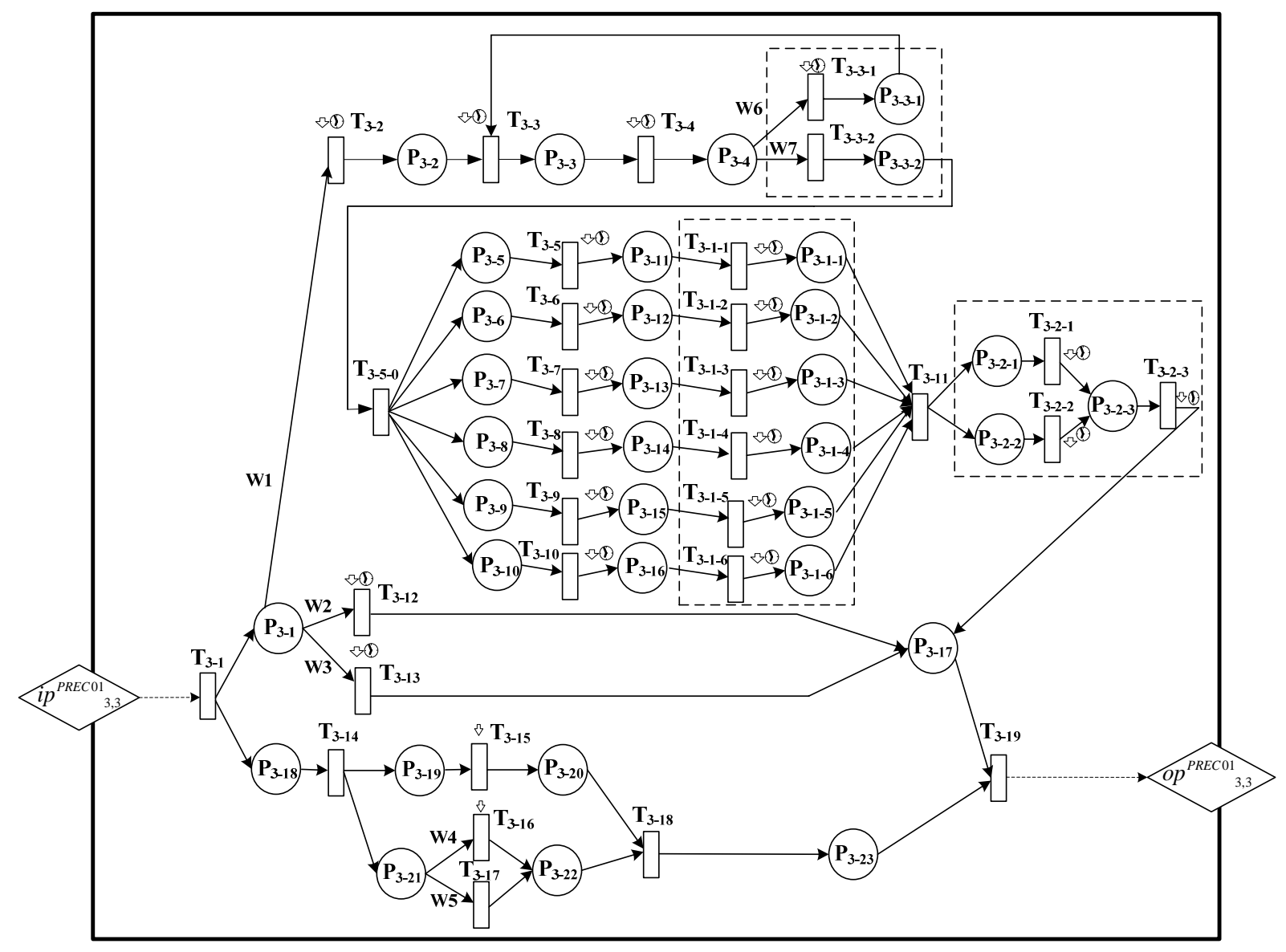

Figure 5: The changed MCPN-CS model of preoperative chemotherapy.

The CBM is formally specified as follows:

$C P N-C_{1,3}=\left(\operatorname{Str}_{1,3}, M_{1,0,3}, M_{0,15,3}, \operatorname{Port}_{1,3}\right), \operatorname{Str}_{1,3}=\left(\sum_{1,3}, P_{1,3}, T_{1,3}, A_{1,3}, C_{1,3}, G_{1,3}, E_{1,3}\right)$; where, the arc set $A_{0,3}$ can be modified by removing arcs $P_{3-11}-T_{3-11}, P_{3-12}-T_{3-11}, P_{3-13}-T_{3-11}$, $P_{3-14}-T_{3-11}, P_{3-15}-T_{3-11}, P_{3-16}-T_{3-11}$ and adding arcs $\boldsymbol{P}_{3-11}-\boldsymbol{T}_{3-1-1}, \boldsymbol{P}_{3-12}-\boldsymbol{T}_{3-1-2}, \boldsymbol{P}_{3-13}-\boldsymbol{T}_{3-1-3}, \boldsymbol{P}_{3-14}-\boldsymbol{T}_{3-1-4}$, ... Therefore, through $A_{1,3}=A_{0,3}-A_{0,3}{ }^{r} \cup A_{0,3}{ }^{a}$, the modified arc set can be got. The result of the modified expression function $E_{0,3}$ can be omitted here. With this model, the CP workflow can be continuous simulated or controlled by the following occurrence sequence:

$$
M_{1,0,3}\left[\left(Y_{1,1,3}, Y_{1,2,3}, Y_{1,3,3}, Y_{1,4,39}, Y_{1,5,3}, Y_{1,6,3}\right)\right]>M_{1,6,3}
$$

$Y_{1,1,3}=\left(t_{3-1-1},<P_{1}>\right), Y_{1,2,3}=\left(t_{3-1-2},<P_{1}>\right), Y_{1,3,3}=\left(t_{3-1-3},<P_{1}>\right), Y_{1,4,3}=\left(t_{3-1-4},<P_{1}>\right), Y_{1,5,3}=\left(t_{3-1-5}\right.$, $\left.<P_{1}>\right), Y_{1,6,3}=\left(t_{3-1-6},<P_{1}>\right), M_{1,6,3}=\left(p_{3-1-1}, P_{1}\right)+\left(p_{3-1-2}, P_{1}\right)+\ldots$

Table IV gives the steps and the corresponding disposing measures for the variations of "HDMTX+CF" chemotherapy scheme. However, at this state, doctors find that only the "high-dose methotrexate (HDMTX) + calcium folinate (CF) rescue" is not an ideal chemotherapy scheme for P1. Therefore, the new osteosarcoma preoperative chemotherapy 
scheme should be adopted. The "(ifosfamide, IFO) + (epirubicin, E-ADM)" osteosarcoma preoperative chemotherapy scheme is an effective method to shrink tumor. So it is necessary to change the CP workflow modelling in time. From Fig. 5 and Table V, shared places and shared transitions between $M C P N C S_{1,3}$ and $A C P N_{1,3}$ are $S P_{1,3}=S P_{1,3}=\left\{\mathbf{P}_{3-17}\right\}$, and $S T_{1,3}=S T_{1,3}{ }^{\prime}=\left\{\mathbf{T}_{3-11}\right\}$, respectively.

Table IV: The steps and corresponding disposing measures.

\begin{tabular}{|c|c|}
\hline Step & Activity \\
\hline $\mathrm{Y}_{1,1}=\left(\mathrm{t}_{3-1-1},<\mathrm{P} 1>\right)$ & Adding the activity of injection G-CSF \\
\hline $\mathrm{Y}_{1,2}=\left(\mathrm{t}_{3-1-2},<\mathrm{P} 1>\right)$ & Ganlixin should be applied \\
\hline $\mathrm{Y}_{1,3}=\left(\mathrm{t}_{3-1-3},<\mathrm{P} 1>\right)$ & Added sodium bicarbonate, to maintain urine $\mathrm{pH}>7$ \\
\hline $\mathrm{Y}_{1,4}=\left(\mathrm{t}_{3-1-4},<\mathrm{P} 1>\right)$ & The oral ulcer ointment and CF solution are applied \\
\hline $\mathrm{Y}_{1,5}=\left(\mathrm{t}_{3-1-5},<\mathrm{P} 1>\right)$ & The ondansetron hydrochloride is injected \\
\hline $\mathrm{Y}_{1,6}=\left(\mathrm{t}_{3-1-6},<\mathrm{P} 1>\right)$ & $\begin{array}{c}\text { The Hydrocortisone, high-dose intravenous glucose and sodium chloride } \\
\text { are injected }\end{array}$ \\
\hline $\mathrm{Y}_{1,7}=\left(\mathrm{t}_{3-11},<\mathrm{P} 1>\right)$ & The treatment for is variation finished \\
\hline $\mathrm{Y}_{1,8}=\left(\mathrm{t}_{3-19},<\mathrm{P} 1>\right)$ & Completing of the preoperative chemotherapy \\
\hline
\end{tabular}

Table V: Interpretation of added places and transitions for the new chemotherapy scheme.

\begin{tabular}{|c|c|c|c|}
\hline Place & Description & Transition & Description \\
\hline $\mathrm{P}_{3-2-1}$ & Preparing for IFO dripping & $\mathrm{T}_{3-2-1}$ & IFO dripping \\
\hline $\mathrm{P}_{3-2-2}$ & Preparing for Mesna injection & $\mathrm{T}_{3-2-2}$ & Mesna injection \\
\hline $\mathrm{P}_{3-2-3}$ & Preparing for E-ADM & $\mathrm{T}_{3-2-3}$ & E-ADM dripping for the eighth day \\
\hline
\end{tabular}

By CBC algorithm, we compose $A C P N_{1,3}$ into $M C P N-C S_{1,3}$ through $S P_{1,3}\left(S P_{1,3}{ }^{\prime}\right)$ and $S T_{1,3}$ $\left(S T_{1,3}\right)$ to obtain the changed $M C P N-C S_{2,3}$ with a new structure. That is:

$$
\begin{aligned}
& \operatorname{Str}_{2,3}=C B C\left(\operatorname{Str}_{1,3}, A C P N_{1,3}, M_{1,6,3}\right)=\left(\sum_{2,3}, P_{2,3}, T_{2,3}, A_{2,3}, C_{2,3}, G_{2,3}, E_{2,3}\right) \text {, and } \\
& M C P N-C S_{2,3}=\left(\operatorname{Str}_{2,3}, M_{2,3}, M_{1,6,3}, \text { Port }_{2,3}\right) ;
\end{aligned}
$$

$M C P N-C S_{2,3}$ is shown in Fig. 5. Among of this, $A_{2,3}, E_{1,3}, P_{2,3}, T_{2,3}$ can be obtained by the same way. With this model, we may continue to simulate or control the osteosarcoma preoperative chemotherapy CP. By the following occurrence sequence (it may not be unique), the case is finished:

$$
\begin{gathered}
M_{2,0,3}\left[Y_{2,1,3}, Y_{2,2,3}, Y_{2,3,3}\right]>M_{2,3,3} \\
Y_{2,1,3}=\left(t_{3-2-1},<P_{1}>\right), Y_{2,2,3}=\left(t_{3-2-2},<P_{1}>\right), Y_{2,3,3}=\left(t_{3-2-3},<P_{1}>\right) \text {, Where } M_{2,3,3}=\left(P_{3-17, P 1}\right)+\ldots
\end{gathered}
$$

Similarly, we can adopt the similar method for MTX concentration detection. Suppose after MTX infusion, the patient's MTX concentration is under the condition of $24 \mathrm{~h}>10$ $\mu \mathrm{mol} / \mathrm{L}$ or $48 \mathrm{~h}>1 \mu \mathrm{mol} / \mathrm{L}$ or $72 \mathrm{~h}>0.1 \mu \mathrm{mol} / \mathrm{L}$. Therefore, the CF dose for chemotherapy will be increased from $12 \mathrm{mg}$ to $15 \mathrm{mg}$. For this, we can adopt CBC algorithm to modify the model.

The additional $A C P N_{2,3}$ is shown in Fig. 5. The shared places and shared transitions between $M C P N-C S_{2,3}$ and $A C P N_{2,3}$ are $S P_{2,3}=S P_{2,3}{ }^{\prime}=\left\{\mathbf{P}_{3-4}\right\}$, and $S T_{2,3}=S T_{2,3}{ }^{\prime}=\left\{\mathbf{T}_{3-5-\mathbf{0}}\right\}$, respectively. By CBC, we compose $A C P N_{2,3}$ into $M C P N-C S_{2,3}$ through $S P_{2,3}\left(S P_{2,3}\right)$ and $S T_{2,3}$ $\left(S T_{2,3}\right)$ to obtain the changed $M C P N-C S_{3,3}$ with a new structure. That is: 


$$
\begin{aligned}
& \operatorname{Str}_{3,3}=C B C\left(\operatorname{Str}_{2,3}, A C P N_{2,3}, M_{2, c, 3}\right)=\left(\sum_{3,3}, P_{3,3}, T_{3,3}, A_{3,3}, C_{3,3}, G_{3,3}, E_{3,3}\right) \text {, and } \\
& M C P N-C S_{3,3}=\left(\operatorname{Str}_{3,3}, M_{3,0,3}, M_{2, c, 3}\right) ;
\end{aligned}
$$

$M C P N-C S_{3,3}$ is shown in Fig. 5 and Table VI. The CBC process for composing the $A C P N_{2,3}$ into $M C P N-C S_{2,3}$ are exactly the same as the above Examples, the details of the CBC process are omitted here. The above steps and their corresponding control measures may lead to final marking, which indicates the completion of the osteosarcoma preoperative chemotherapy.

By this way, these variations of the osteosarcoma preoperative chemotherapy can be described clearly. Moreover, based on the initial model and the changed model, the occurrence sequence of transitions can be obtained. This occurrence sequence of transitions demonstrates this method is feasible.

Table VI: Interpretation of added places and transitions of MTX concentration detecting.

\begin{tabular}{|c|c|c|c|}
\hline Place & Description & Transition & Description \\
\hline $\mathrm{P}_{3-3-1}$ & $\begin{array}{c}\text { The patient's MTX concentration } \\
\text { is abnormal and preparing for } \\
\text { increasing CF dose from } 12 \mathrm{mg} \text { to } \\
15 \mathrm{mg}\end{array}$ & $\mathrm{T}_{3-3-1}$ & $\begin{array}{c}\text { Detecting the patient's MTX } \\
\text { concentration is under the condition } \\
\text { of } 24 \mathrm{~h}>10 \mu \mathrm{mol} / \mathrm{L} \text { or } 48 \mathrm{~h}>1 \\
\mu \mathrm{mol} / \mathrm{L} \text { or } 72 \mathrm{~h}>0.1 \mu \mathrm{mol} / \mathrm{L}\end{array}$ \\
\hline $\mathrm{P}_{3-3-2}$ & $\begin{array}{c}\text { The patient's MTX concentration } \\
\text { is normal }\end{array}$ & $\mathrm{T}_{3-3-2}$ & Dumb activity \\
\hline
\end{tabular}

\subsection{Structural change of the whole Workflow model and the deadlock detection}

As in Section 3.2 shown, the patient A.1 is treated. According to Figs. 2 and 3, before the preoperative chemotherapy, the stage of osteosarcoma patient A.1 is the II B stage. Therefore, its treatment scheme is "Preoperative Chemotherapy + Imaging and Clinical Evaluation + Making Surgery Scheme1+ Amputation Operation + Postoperative Evaluation and Treatment”. However, after the preoperative chemotherapy, patient A.1 presents the lung metastases. Therefore, the staging of osteosarcoma will be turned into the III B. Therefore treatment scheme is changed into "Make Surgery Scheme2 + Resection of Lung Metastases + Radical Amputation Surgery + Adjuvant Therapy". This changed information will be loaded the MBG dynamically. That is to say, the structural changes of the whole workflow model can be realized by the changed relationships of different modules through the MBG. In the same way, DDA can determine whether the MCPN-CS model is live and deadlock-free. Therefore, the whole changed MCPN-CS model will be concluded live and deadlock-free.

\section{CONCLUSION}

A Modular Coloured Petri Net with changeable structures (MCPN-CS) workflow modelling method is proposed. Aimed at the variations of the CP, the evolution workflow model for the $\mathrm{CP}$ may be reconfigured dynamically by using the mechanisms of change-by-modification (CBM) and change-by-composition (CBC). A case study is given to show how to build the MCPN-CS-based reconfigurable workflow model for the osteosarcoma CP and analyze the reconfigurable model by presented deadlock detection algorithms. The results show that the proposed method can enhance the flexibility and adaptation of the CP workflow modelling noticeably.

Moreover, MCPN-CS approach may be applied in performance analysis, optimization, simulation, and adaptive scheduling and control of the CP. Possible future research may focus on the MCPN-CS-based adaptive scheduling and control method of the CP. 


\section{ACKNOWLEDGEMENTS}

This work was supported by Research Grant from National Natural Science Foundation of China (60774103) and Major Program Development Fund of SJTU. Moreover, we would like to thank the whole medical staff of Shanghai No. 6 People's Hospital for helpful discussions. We also wish to thank the journal editor and the referees for helpful suggestions to improve the paper.

\section{REFERENCES}

[1] Bragato, L.; Jacobs, K. (2003). Care pathways: the road to better health services, Journal of Health Organization and Management, Vol. 17, No. 3, 164-180

[2] Cheah, J. (2000). Development and implementation of a clinical pathway programme in an acute care general hospital in Singapore, International Journal for Quality in Health Care, Vol. 12, No. $5,403-412$

[3] Hunter, B.; Segrott, J. (2008). Re-mapping client journeys and professional identities: A review of the literature on clinical pathways, International Journal of Nursing Studies, Vol. 45, No. 4, 608-625

[4] Wakamiya, S. J.; Yamauchi, K. (2009). What are the standard functions of electronic clinical pathways? International Journal of Medical Informatics, Vol. 78, No. 8, 543-550

[5] Kealey, G. P.; Burger, M. (1995). Utilization of critical pathways to facilitate clinical care, The Journal of Burn Care Rehabilitation, Vol. 16, No. 22, 214-216

[6] Workflow Management Coalition. (1995). Workflow Reference Model, Doc.TC00-1003, Iss.1.1. http://www.aiim.org/wfmc/mainframe.htm

[7] Dwivedi, A.; Bali, R. K.; James, A. E.; Naquib, R. N. (2001). Workflow management systems: the healthcare technology of the future Annual, Reports of the Research Reactor Institute, Vol. 4, 3887-3890

[8] Wainer, J.; de Lima Bezerra, F. (2003). Constraint-Based Flexible Workflows. CRIWG, LNCS Vol. 2806, 151-158, Springer-Verlag, Berlin

[9] Bricon-Souf, N.; Renard, J. M.; Beuscart, R. (1999). Dynamic workflow model for complex activity in intensive care unit, International Journal of Medical Informatics, Vol. 53, 143-150

[10] Ye, Y.; Jiang, Z. B.; Diao, X. D.; Yang, D.; Du, G. (2009). An ontology-based hierarchical semantic modeling approach to clinical pathway workflows, Computers in Biology and Medicine, Vol. 39, No. 8, 722-73

[11] Van der Aalst, W. M. P.; Van Hee, K. M.; Houben, G. J. (1994). Modelling and analysing workflow using a Petri-net based approach, Proceedings of the Second Workshop on Computer-Supported Cooperative Work, Petri nets and Related Formalisms, 31-50

[12] Van der Aalst, W. M. P. (1998). The Application of Petri Nets to Workflow Management, The Journal of Circuits, Systems and Computers, Vol. 8, No. 1, 21-66

[13] Garelli, M.; Gribaudo, M. (2009). Performance Analysis of the ARIA Adaptive Media Processing Workflows using Colored Petri Nets, Electronic Notes in Theoretical Computer Science, Vol. 232, No. 26, 55-73

[14] Ha, S.; Suh, H. W. (2008). A timed colored Petri nets modeling for dynamic workflow in product development process, Computers in Industry, Vol. 59, No. 2-3, 193-209

[15] Liu, D.; Wang, J.; Chan, S. C. F. (2002). Modeling workflow processes with colored Petri nets, Computers in Industry, Vol. 49, 267-281

[16] Fung, R. Y. K.; Au, A. Y. M.; Jiang, Z. B. (2003). Supply chain workflow modelling using XML-formatted modular Petri nets, The International Journal of Advanced Manufacturing Technology, Vol. 22, No. 7-8, 587-601

[17] Jiang, Z. B.; Zuo, M. J.; Fung, R. Y. K.; Tu, P. Y. (2001). Colored Petri Nets with changeable structures (CPN-CS) and their applications in modeling one-of-a-kind production (OKP) systems, Computers \& Industrial Engineering, Vol. 41, No. 3, 279-307

[18] Jiang, Z. B.; Zuo, M. J.; Fung, R. Y. K.; Tu, P. Y. (2000). Temporized coloured Petri nets with changeable structure (CPN-CS) for performance modelling of dynamic production systems, 
Du, Jiang, Diao, Yao: Reconfigurable Modelling with Deadlock Avoidance for the Clinical ...

International Journal of Production Research, Vol. 38, No. 8, 1917- 1945.

[19] Jensen, K. (1992). Colored Petri Nets: Basic Concepts, Analysis Methods and Practical Use, Vol. 1, Springer, New York

[20] Jensen, K. (1995). Colored Petri Nets: Basic Concepts, Analysis Methods and Practical Use, Vol. 2, Springer, New York

[21] Liu, H. R.; Jiang, Z. B.; Fung, R. Y. K. (2004). An Extended Object-oriented Petri Nets for Modeling and Analysis Automated Re-entrant Manufacturing Systems, IEEE International Conference on Systems, Man and Cybernetics, 1654-1659

[22] D'souza, K. A. (1994). A Control Model for Detecting Deadlocks in an Automated Machining Cell, Computers \& Industrial Engineering, Vol. 26, 133-139 